\title{
Conformal and Smooth TiN Film Growth by using Variable-Pressure Thermal ALD Method
}

\author{
Hyunchol Cho ${ }^{1}$, Sung-Hoon Jung ${ }^{1}$, Ben $\mathrm{Nie}^{1}$, Anu Nair ${ }^{1}$, Zhigang Chen ${ }^{1}$, Somilkumar \\ Rathi $^{1,2}$ and Niloy Mukherjee ${ }^{1}$ \\ ${ }^{1}$ Eugenus, Inc., 677 River Oaks Parkway, San Jose, CA, USA, 95134 \\ ${ }^{2}$ Department of Materials Science and Engineering, University of Central Florida, \\ Orlando, FL, USA, 32816
}

\begin{abstract}
Better TiN film quality such as lower roughness and higher step coverage is required in recent semiconductor devices as the semiconductor device is scaled down. In this study, highly conformal ALD TiN film with excellent step coverage was achieved by using variable-pressure deposition method. TiN film grown at low-pressure condition was more conformal and smoother than that grown at high-pressure condition, although step coverage value grown at low-pressure condition was worse than that grown at high-pressure condition. By optimizing low/high pressure two-step TiN growth condition, it was achieved that not only better roughness than that of pure high-pressure TiN, but also, better step coverage value than that of pure low-pressure TiN as well as than that of pure high-pressure TiN.
\end{abstract}

\section{Introduction}

As the generation of memory devices evolve, the successful fabrication of high aspect ratio (HAR) features becomes more and more challenging. Apart from the traditional patterning, deposition and etch related issues, conformal film deposition onto these HAR structures becomes a critical parameter in determining the overall device yields. It is well established now that compared to conventional deposition techniques such as chemical vapor deposition $(\mathrm{CVD})^{1,2}$ and physical vapor deposition $(\mathrm{PVD})^{3}$, atomic layer deposition (ALD) $)^{4,5}$ offers a pathway to highest conformality and step-coverage. On the other hand, film conformality, especially roughness property has trade-off relationship to step-coverage property. High-pressure process is appropriate to obtain high step-coverage performance in short ALD cycle time process, but roughness value becomes worse due to higher chance of crystalline film growth at high-pressure process condition. High roughness causes local variations in step-coverage and poses problems for deposition of subsequent layers and device performance.

\section{Experimental}

In the present study, we report that it is possible to obtain conformal and smooth TiN film by using low/high pressure two-step growth, initially low-pressure TiN growth, and secondly high-pressure TiN growth on the non-pattern and HAR pattern wafers. Eugenus's versatile $300 \mathrm{~mm}$ mini-batch system and HAR structures with approximately 
120:1 (Dimension of bottom opening, and total height are approximately $17 \mathrm{~nm}$ and $2 \mu \mathrm{m}$, respectively) were used in this research, respectively. $\mathrm{TiCl}_{4}$ and $\mathrm{NH}_{3}$ were used as the precursors for TiN film deposition and grown TiN films were analyzed by using ellipsometry, atomic force microscopy (AFM) and transmission electron microscopy (TEM) to study the film properties and step-coverages.

\section{Results and Discussion}

Initially, various pressure condition was tested in terms of growth per cycle (GPC) and GPC value was increased by using higher chamber pressure condition, as shown in Figure 1. This phenomenon is due to higher chance of precursor resident time when high pressure condition was used for the deposition.

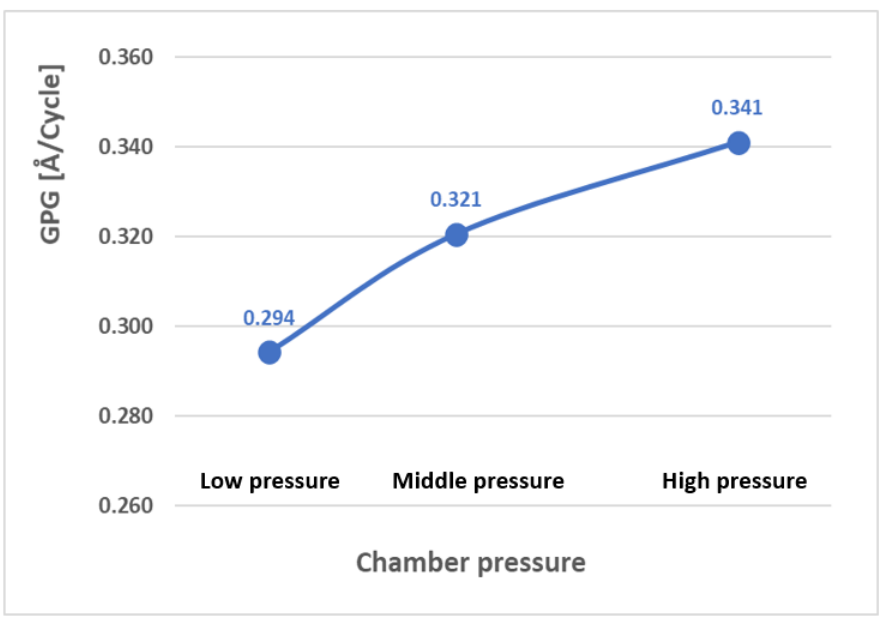

Figure 1. TiN growth per cycle (GPC) by using different chamber pressure condition.

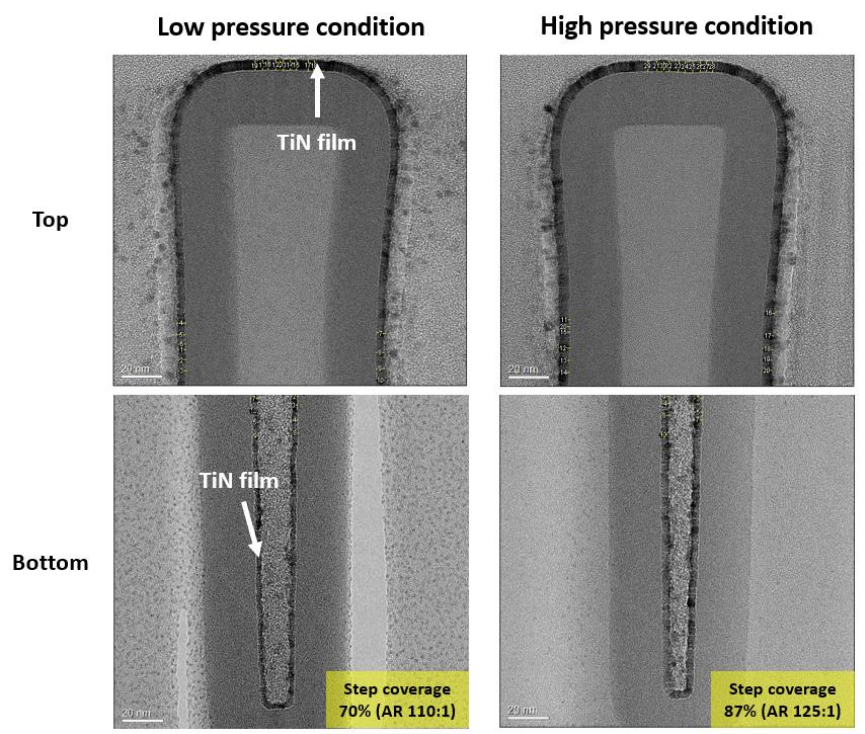

Figure 2. TEM images of TiN film grown on the pattern by using high pressure condition. 
It was confirmed that TiN film grown at low-pressure condition was more conformal and smoother than that grown at high-pressure condition, although step coverage value grown at low-pressure condition was worse than that grown at high-pressure condition, as shown in Figure 2. We are using bottom side thickness and top side thickness for the calculation of the step coverage value, as shown in Equation [1].

$$
\frac{\text { Bottom side thickness }}{\text { Top side thickness }} * 100 \quad \text { Equation [1] }
$$

It was tried to adapt the low/high pressure two-step growth to obtain better roughness and step coverage value. Initial low-pressure deposition cycle was varied from $0 \%$ to $100 \%$ and checked Root Mean Square (RMS) value by AFM analysis, as well as step coverage value by TEM analysis. By adapting approximately $10 \%$ of low-pressure deposition cycle in total deposition cycle, it was possible to achieve that not only better roughness than that of pure high-pressure TiN, but also, better step coverage value than that of pure low-pressure TiN as well as than that of pure high-pressure TiN (Figure 3). This result is indicating that the initial smooth (2-dimensional) TiN film growth is affecting to the step coverage property. In order to understand the underlying fundamental mechanisms of the pressure effect on surface roughness and step coverage, we are conducting both macroscopic computational fluid dynamics (CFD) simulations and microscopic kinetic simulations to establish connections between precursor residence time, mass transport and diffusion and experimental data.

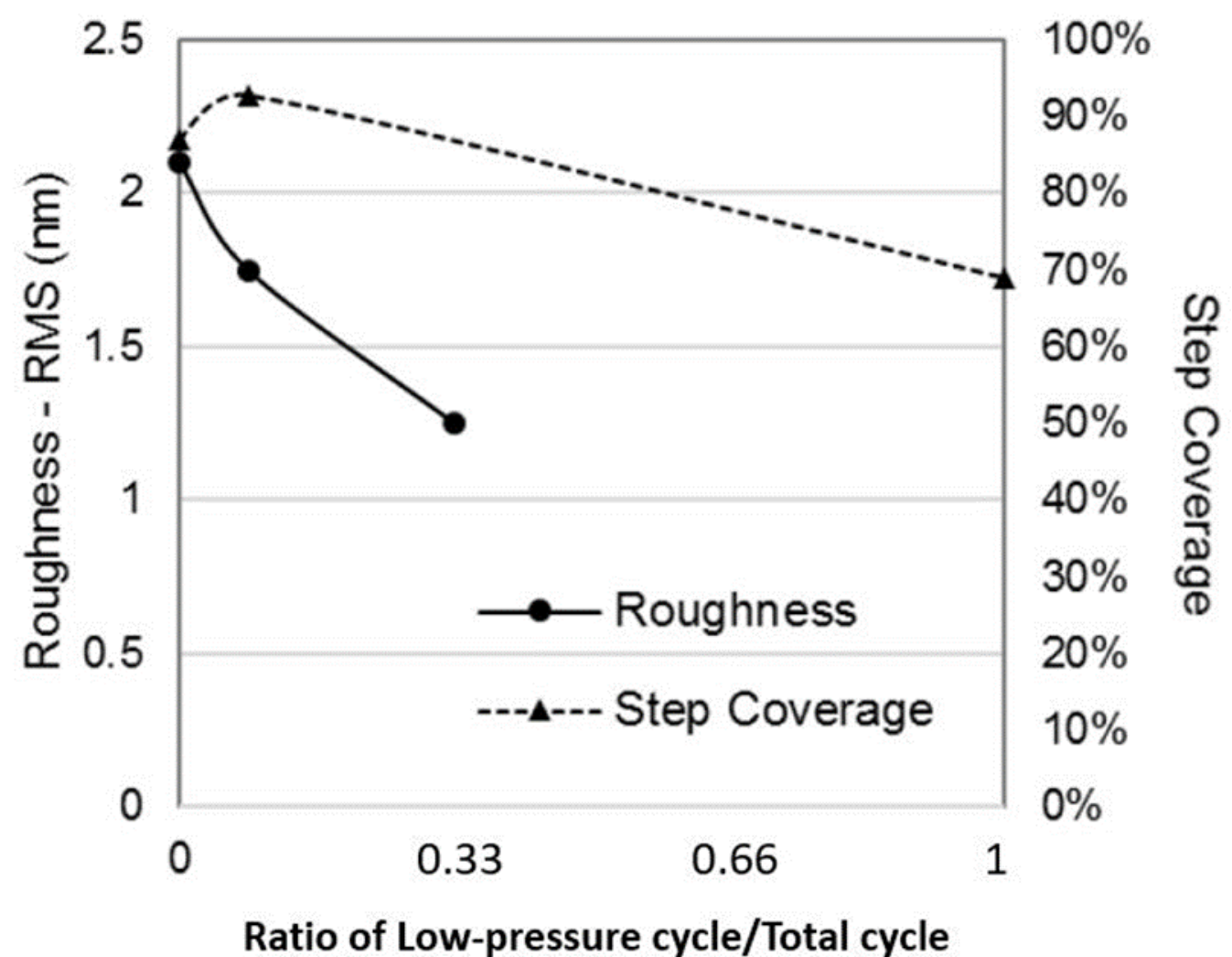

Figure 3. AFM roughness and step coverage data by using two-step growth method. 


\section{Conclusions}

Conformal and smooth TiN film was grown by using variable-pressure thermal ALD method. It was possible to obtain TiN film having better roughness and step coverage value by adapting approximately $10 \%$ of low-pressure deposition cycle in total deposition cycle than that grown by using pure high pressure one step method.

\section{References}

1. Dae-Gyu Park, Heung-Jae Cho, Kwan-Yong Lim, Tae-Ho Cha, In-Seok Yeo and Jin Won Park, Journal of The Electrochemical Society, Volume 148, Number 9 (2001) F189.

2. Ming-Da Cheng, Tuung Luoh, Chin-Ta Su, Ta-Hung Yang, Kuang-Chao Chen and Chih-Yuan Lu, Thin Solid Films, Volume 518, Issue 8, (2010) 2285-2289.

3. Yongxun Liu, Takahiro Kamei1, Kazuhiko Endo, Shinichi O'uchi, Junichi Tsukada, Hiromi Yamauchi, Tetsuro Hayashida1, Yuki Ishikawa, Takashi Matsukawa, Kunihiro Sakamoto, Atsushi Ogura1 and Meishoku Masahara, Japanese Journal of Applied Physics, Volume 49, (2010) Number 6S.

4. Jinjuan Xiang, Tingting Li, Xiaolei Wang, Kai Han, Junfeng Li and Chao Zhao, ECS Journal of Solid-State Science and Technology, Volume 5 (2016) P327.

5. M. G. Kozodaev, Y. Y. Lebedinskii, A. G. Chernikova, S. N. Polyakov and A. M. Markeev, Phys. Status Solidi A, 214 (2017) 1700056. 